Short paper

\title{
Type V collagen counteracts osteo-differentiation of human mesenchymal stem cells
}

\author{
Alessandra Longo ${ }^{a}$, Edda Tobiasch ${ }^{\mathrm{b}}$, Claudio Luparello ${ }^{\mathrm{a}, *}$ \\ a Dipartimento di Scienze e Tecnologie Biologiche, Chimiche e Farmaceutiche (STEBICEF), Università di Palermo, Italy \\ ${ }^{\mathrm{b}}$ Department of Natural Sciences, Bonn-Rhein-Sieg University, Rheinbach, Germany
}

\section{A R T I C L E I N F O}

Article history:

Received 5 February 2014

Received in revised form

3 July 2014

Accepted 7 July 2014

Available online $\mathrm{xxx}$

Keywords:

Stem cells

Collagen

Osteogenesis

Gene expression

\begin{abstract}
A B S T R A C T
In search of novel gene signatures for osteo-differentiation of mesenchymal stem cells (MSCs), we submitted cDNA preparations from undifferentiated and differentiating MSCs to differential display- and semiquantitative-PCR and found down-regulation of COL5A1 in osteo-induced cultures at days 21 and 28 , when the mineralized matrix accumulates. We also cultured osteo-differentiating MSCs onto type V collagen substrates and found a decrease in the accumulation of extracellular calcium compared to those grown in uncoated flasks. To our knowledge, this is first evidence that type $\mathrm{V}$ collagen might represent a stromal component that impairs osteogenesis.
\end{abstract}

๑) 2014 The International Alliance for Biological Standardization. Published by Elsevier Ltd. All rights reserved.

\section{Introduction}

Human fat tissue represents a valid alternative source of mesenchymal stem cells (MSCs) to bone marrow (BM), which allows obtaining an in vitro model system of primary cultures containing the stem cell population present in situ. Furthermore, liposuction samples permit the isolation of a 500 times higher number of cells as compared to BM-derived MSCs, avoiding additional pain and discomfort for the patient, without major ethical limitations for their use. The multipotentiality of MSCs from adipose tissue, coupled to their self-renewability, is closely linked to their therapeutic interest, in that these cells might find utilization in cell therapy and tissue engineering, being easily addressed in vitro to differentiate into diverse cytotypes, including osteoblasts (e.g. Refs. [1,2]). Although the differentiation of fat tissue-derived MSCs into various lineages are standard procedures, the precise molecular mechanisms involved are still poorly understood, making the study of the differentiation pathways of these cells of basic

Abbreviations: MSC, mesenchymal stem cell; BM, bone marrow; PCR, polymerase chain reaction; dNTPs, deoxynucleotide triphosphates; DD, differential display.

* Corresponding author. Dipartimento STEBICEF, Università di Palermo, Edificio 16, Viale delle Scienze, 90128 Palermo, Italy. Tel.: +39 09123897405; fax: +39 0916577210.

E-mail addresses: claudio.luparello@unipa.it, clupar@tin.it (C. Luparello). scientific interest to identify new putative markers of stemness, because the transient utilisation of such pathways may allow the amplification of stem cells in culture which represents a current challenge in most cell-based therapies and in the field of regenerative medicine. In addition, it is necessary to have a more detailed knowledge of the differentiation event to determine the impact of new molecules on the process of tissue formation. Here we report that type $\mathrm{V}$ collagen might be considered as a negative stromal modulator of MSC osteogenesis.

\section{Materials and methods}

\subsection{Cell cultures, collagen substrate and differentiation}

LW28, LW36 and LW40 MSC cultures, isolated from adipose tissue samples obtained by liposuction of three female patients, respectively 28, 36 and 40 years old, were differentiated at passage 5 towards osteoblast lineage, and the differentiation checked as already shown by Longo et al. [3].

Type V collagen substrate (Sigma) was prepared as reported in Ref. [4]. MSCs were plated at a concentration of $2 \times 10^{4}$ cells $/ \mathrm{cm}^{2}$ with the osteogenic differentiation medium in plain and collagen-coated dishes. The media were carefully changed once a week. After four weeks, osteogenic differentiation was visualized by staining of extracellular calcium deposits with Alizarin Red S [3]. 


\subsection{Reverse transcription}

Total RNA was obtained with Tri Reagent (Sigma) and enriched mRNA fractions with Terminator ${ }^{\mathrm{TM}}$ 5'-Phosphate-Dependent Exonuclease (Epicentre, Madison, WI/USA). The cDNAs were synthesized using SuperScript II reverse transcriptase (Invitrogen) as reported in Ref. [3].

\subsection{Differential display (DD)- and semiquantitative $P C R$}

DD-PCR experiments were performed using the arbitrary 10mer primers designed by Sokolov and Prockop [5], as reported in Refs. [6,7]. The PCR products were analyzed with 6\% nondenaturing PAGE in a sequencing apparatus followed by silver staining. A 50 bp ladder (Invitrogen) was used as a size marker.
For semiquantitative PCR, COL5A1 was co-amplified with $18 \mathrm{~S}$ cDNA and the relative difference in abundance between the experimental conditions evaluated as reported in Ref. [8].

\section{Results and discussion}

In order to expand the list of gene signatures associated with osteo-induction of MSCs, enriched mRNA samples isolated from undifferentiated and differentiating LW36 cell preparations at day 7, 14, 21 and 28 of culture were reverse transcribed and submitted to differential display-PCR (DD-PCR), and the amplification products electrophoresized and visualized by silver stain. Among the different products present in the gel, we focused our interest on an amplification band of approximately 150 bp (Fig. 1A) selectively absent in the cDNA preparation of MSCs at the third week of
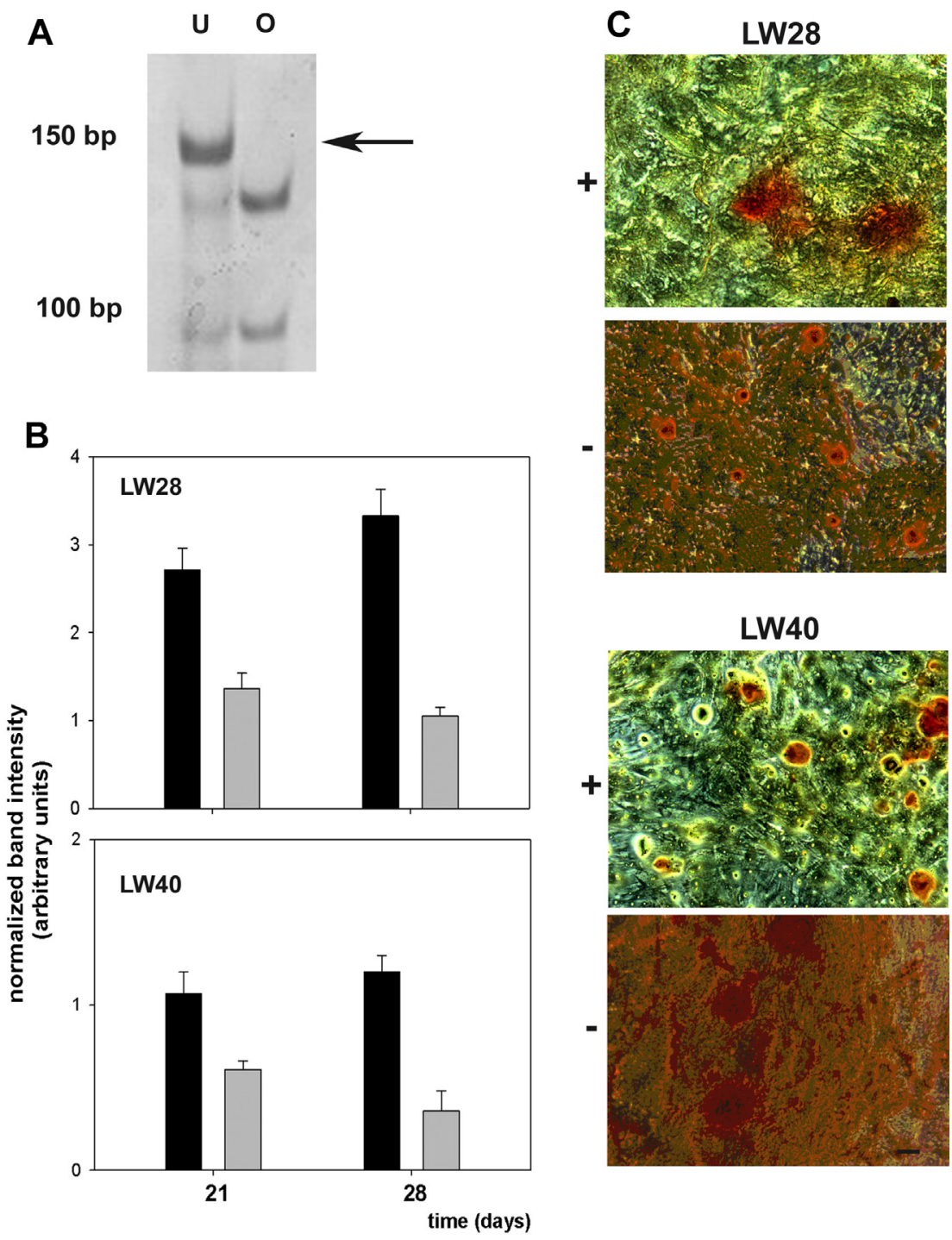

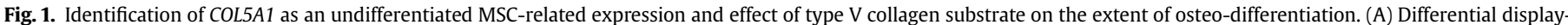

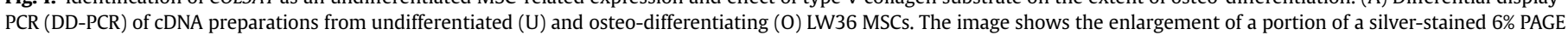

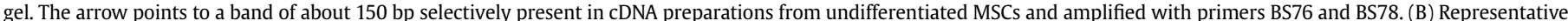

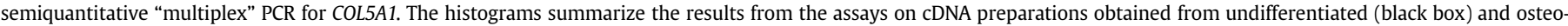

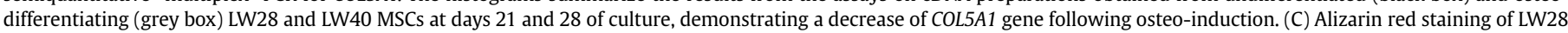

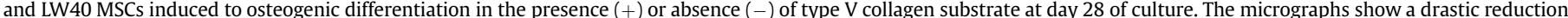

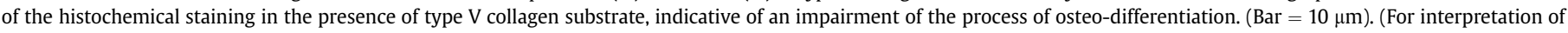
the references to colour in this figure legend, the reader is referred to the web version of this article.) 
differentiation, that is when phenotypic osteogenesis begins to be visible by Alizarine Red histochemical staining of calcium-rich deposits [4]. The cDNA contained in the band was purified by excision from the gel, re-amplified until purity and adequate yield, and then the material was submitted to sequencing. Homology was found in the online databases between the sequence obtained and that of region $5491-5635$ of the coding sequence for $\alpha_{1}$ chain of type $\mathrm{V}$ collagen (COL5A1, gene id: 1289 COL5A1; score: 455 bits, expect: $5 e-126$, identities $100 \%$, gap $0 \%$ ). Interestingly, the analysis by FASTA also showed homology with region $5491-5635$ of a patented sequence called "stem cell specific marker" (acc. nr. DM149324; Zscore: 1176.1 , bits: 230.2 , expect: $1.4 \mathrm{e}-56$, identity $100 \%$, gap $0 \%$ ). For semi-quantitative evaluation in more stringent conditions than DD-PCR and to investigate whether the differential expression was also present in the cell cultures obtained from the other donors, the cDNA preparations from either undifferentiated and differentiating LW28 or LW40 cells at days 21 and 28 of culture were submitted to conventional and multiplex PCR analysis in the presence of COL5A1 specific primers [9], and amplifying the housekeeping gene $18 \mathrm{~S}$ in parallel. The obtained results showed that an amplification signal for COL5A1 was present in all experimental conditions (not shown), but in osteo-induced cells the COL5A1 expression level was downregulated compared to undifferentiated stem cells, to about $50 \%$ and $31.5 \%$ (for LW28 cells) and 57\% and 30\% (for LW40 cells) at days 21 and 28 of culture, respectively, that is when the mineralized matrix accumulates (Fig. 1B). No difference of COL5A1 expression level could be observed between stem and differentiating cells at days 7 and 14 of culture (not shown). No attempt was made to quantify the produced type $\mathrm{V}$ collagen in the different experimental conditions.

Secondly, assuming that the down-regulation of COL5A1 might be essential to the acquisition of a mature osteogenic phenotype by differentiating cells, flasks covered with bi-dimensional type $\mathrm{V}$ collagen films were prepared to investigate whether this collagen, used as a substrate for MSC cultures submitted to exposition to osteogenic inducers, could restrain the differentiation process, due to the continuous and prolonged adhesion of MSCs. Osteogenesis was monitored through the deposition of extracellular calcium by Alizarin Red staining. The data obtained at the fourth week of differentiation showed a drastic decrease in the accumulation of extracellular calcium by LW28 and LW40 cells, compared to the counterparts induced to differentiation in the absence of type $\mathrm{V}$ collagen substrate (Fig. 1C).

It is generally acknowledged that the extracellular matrix (ECM) is not to be regarded as a mere passive scaffold for connective tissues within organ architecture, but that cell-ECM interactions may influence a number of biological activities. Collagen, the major constituents of connective tissues, is a protein family composed of at least 28 genetically-distinct species, grouped in different categories, originating from the coiling of three polypeptides of about 1000 aminoacids called $\alpha$-chains. The $\alpha$-chain composition of the different collagens can give rise to either homo- or heterotrimeric types that accumulate in the ECM, thereby interacting with other micro-environmental components as well as cell receptors, thereby exerting a prominent feedback effect on the control of cell phenotypic expression [10]. Type V collagen, which belongs to the category of the fibril-forming collagens and may be assembled as $\left[\alpha_{1}(\mathrm{~V})\right]_{2} \alpha_{2}(\mathrm{~V}), \alpha_{1}(\mathrm{~V})_{3}$ and $\alpha_{1}(\mathrm{~V}) \alpha_{2}(\mathrm{~V}) \alpha_{3}(\mathrm{~V})$ variants, is a minor component of the stroma which can be predominantly found in pericellular areas [11,12]. The cellular responses elicited by this collagen species appear heterogeneous, suggesting differences among the model systems examined (e.g. tissue and cell types). For example, adhesion- and motility-promoting activity has been observed for glomerular endothelial cells, Schwann cells, and osteosarcoma cells [13-15]. On the other hand, anti-adhesive, anti- proliferative and/or anti-locomotory effects have been observed on cytotypes such as fibroblasts, colorectal adenocarcinoma cells, and lung epithelial cells [16-18]. In addition, a putative "anti-cancer" activity has been found when used as a substrate for 8701-BC breast cancer cells, determining slow-down of growth rate, inhibition of motility and invasion in vitro, loss of membrane integrity, oligonucleosomal DNA fragmentation, changes in the expression levels of genes coding for apoptosis-linked factors, calpain, caspases and stress response proteins, and enhancement of caspase enzymatic activity $[4,8,19-21]$. The data reported here expand the list of the recognized cellular effects of type $\mathrm{V}$ collagen, being, to our knowledge, the first evidence that this collagen species, whose $\alpha_{1}$ chain is encoded by a gene that in the databases is also defined "stem cell marker", might represent a component of the extracellular matrix that counteracts the effect of osteogenic factors on MSCs. Interestingly, Fawzy El-Sayed and co-workers [22] reported that STRO-1-expressing multipotent postnatal stem/progenitor cells [23] isolated from human alveolar bone displayed a significantly-higher expression of mineralized tissue markers, including type $\mathrm{V}$ collagen, with respect to periodontal osteoblasts extracted from the same source.

\section{Conclusions}

In conclusion, our results expand the list of the microenvironmental components controlling the process of bone formation and prompt the setting up of future experiments aimed to investigate more in detail the cellular and molecular aspects of the inhibitory effect shown by type $\mathrm{V}$ collagen substrate on osteodifferentiation of MSCs, thereby extending the knowledge about the intracellular processes that impair the differentiation event. Technical-scientific advances in the areas of cellular and molecular biology, facilitated by studies about human somatic stem cells, may positively influence the advance of clinical research with MSCs. The knowledge of the basic mechanisms of differentiation may thus help the development of new therapies and the evaluation of biosecurity issues in future clinical protocols.

\section{Acknowledgments}

The work was supported by University of Palermo (R.S. ex60\% and FFR 2013) and Progetto Vigoni 2011 for Claudio Luparello, and BMBF, AdiPaD, 1720X06, FHprofUnt, FKZ: 03FH012PB2; FH-Extra, Europäischer Fonds für regionale Entwicklung, Europa - Investition in unsere Zukunft, FKZ: z1112fh012; DAAD, PPP Vigoni, FKZ: 54669218; BMBF-AIF, FKZ: 1720X06; and Geräteprogramm, Fachhochschulen NRW 2008 for Edda Tobiasch.

\section{References}

[1] Khan WS, Hardingham TE. Mesenchymal stem cells, sources of cells and differentiation potential. J Stem Cells 2012;7:75-85.

[2] Zhang Y, Khan D, Delling J, Tobiasch E. Mechanisms underlying the osteo- and adipo-differentiation of human mesenchymal stem cells. ScientificWorldJournal 2012:793823.

[3] Longo A, Librizzi M, Naselli F, Caradonna F, Tobiasch E, Luparello C. PTHrP in differentiating human mesenchymal stem cells: transcript isoform expression, promoter methylation, and protein accumulation. Biochimie 2013;95: $1888-96$.

[4] Luparello C, Sirchia R. Type V collagen-induced up-regulation of capn2 (large subunit of $\mathrm{m}$-calpain) and DNA fragmentation in 8701-BC breast cancer cells. Biol Chem 2011;392:501-4.

[5] Sokolov BP, Prockop DJ. A rapid and simple PCR-based method for isolation of cDNAs from differentially expressed genes. Nucleic Acids Res 1994;22: 4009-15.

[6] Longo A, Librizzi M, Luparello C. Effect of transfection with PLP2 antisense oligonucleotides on gene expression of cadmium-treated MDA-MB231 breast cancer cells. Anal Chem Bioanal Chem 2013;405:1893-901.

[7] Luparello C, Sirchia R, Longo A. Type V collagen and protein kinase C $\eta$ downregulation in 8701-BC breast cancer cells. Mol Carcinog 2013:52:348-58. 
[8] Luparello C, Sirchia R. Type V collagen regulates the expression of apoptotic and stress response genes by breast cancer cells. J Cell Physiol 2005;202: 411-21.

[9] Luna C, Li G, Qiu J, Epstein DL, Gonzalez P. Role of miR-29b on the regulation of the extracellular matrix in human trabecular meshwork cells under chronic oxidative stress. Mol Vis 2009;15:2488-97.

[10] Gelse K, Pöschl E, Aigner T. Collagens - structure, function, and biosynthesis. Adv Drug Deliv Rev 2003;55:1531-46.

[11] Birk DE. Type V collagen: heterotypic type I/V collagen interactions in the regulation of fibril assembly. Micron 2001;32:223-37.

[12] Luparello C. Aspects of collagen changes in breast cancer. J Carcinog Mutagene 2013;:S13:007.

[13] Chernousov MA, Stahl RC, Carey DJ. Schwann cell type V collagen inhibits axonal outgrowth and promotes Schwann cell migration via distinct adhesive activities of the collagen and noncollagen domains. J Neurosci 2001;21: 6125-35.

[14] Yamaguchi K, Matsuo N, Sumiyoshi H, Fujimoto N, Iyama KI, Yanagisawa S, et al. Pro-alpha3(V) collagen chain is expressed in bone and its basic Nterminal peptide adheres to osteosarcoma cells. Matrix Biol 2005;24: 283-94.

[15] Murasawa Y, Hayashi T, Wang PC. The role of type V collagen fibril as an ECM that induces the motility of glomerular endothelial cells, Exp. Cell Res 2008;314:3638-53.
[16] Parekh T, Wang X, Makri-Werzen DM, Greenspan DS, Newman MJ. Type V collagen is an epithelial cell cycle inhibitor that is induced by and mimics the effects of transforming growth factor ß1. Cell Growth Differ 1998;9:423-33.

[17] Köbel M, Budianto D, Schmitt WD, Borsi L, Siri A, Hauptmann S. Influence of various cytokines on adhesion and migration of the colorectal adenocarcinoma cell line HRT-18. Oncology 2005;68:33-9.

[18] Breuls RG, Klumpers DD, Everts V, Smit TH. Collagen type V modulates fibroblast behavior dependent on substrate stiffness. Biochem Biophys Res Commun 2009:380:425-9.

[19] Luparello C, Schillaci R, Pucci-Minafra I, Minafra S. Adhesion, growth and cytoskeletal characteristics of 8701-BC breast carcinoma cells cultured in the presence of type V collagen. Eur J Cancer 1990;26:231-40.

[20] Luparello C, Sheterline P, Pucci-Minafra I, Minafra S. A comparison of spreading and motility behaviour of 8701-BC breast carcinoma cells on type I, I-trimer and type V collagen substrata. J Cell Sci 1991;100:179-85.

[21] Luparello C. Adhesion to type V collagen and cloning efficiency in agar of 8701-BC breast cancer cells. Eur J Cancer 1994;30:1400-1.

[22] Fawzy El-Sayed KM, Paris S, Becker S, Kassem N, Ungefroren H, Fändrich F, et al. Isolation and characterization of multipotent postnatal stem/progenitor cells from human alveolar bone proper. I Craniomaxillofac Surg 2012:40:735-42.

[23] Gronthos S, Graves SE, Ohta S, Simmons PJ. The STRO-1+ fraction of adult human bone marrow contains the osteogenic precursors. Blood 1994;84 4164-73. 